\title{
Tuberculosis control is good for established leprosy programmes
}

\author{
ROSEMARY A. CROFT \& RICHARD P. CROFT \\ The Leprosy Mission, Danish Bangladesh Leprosy Mission, PO Box 3, \\ PO and District Nilphamari 5300, Bangladesh
}

Accepted for publication 11 November 1996

\begin{abstract}
Summary Tuberculosis (TB) control was introduced into part of the Danish Bangladesh Leprosy Mission's large leprosy control programme in 1994. This was in line with the Government's policy of combining leprosy and TB control. We report our experience with integration. Leprosy case-finding has increased during the period, and staff satisfaction and morale has also risen despite the larger workload. We observed that the field work skills of leprosy workers was brought to bear in a very positive way on TB control. TB patients suffer considerable impoverishment as a result of their illness, paralleling the social dehabilitation often seen in leprosy sufferers. TB control is good for established leprosy programmes.
\end{abstract}

\section{Introduction}

Combining tuberculosis control with leprosy control has often been suggested as a way forward for vertical leprosy programmes to sustain their services in situations of falling prevalence. ${ }^{1,2}$ In a recent article in the British Medical Journal, Lockwood \& Saunderson ${ }^{3}$ drew attention to the way that the traditional strengths of leprosy programmes - namely in case-holding and case-finding — can be successfully harnessed to TB control to the mutual benefit of both services. They were writing from the background of a successful programme at ALERT, Ethiopia where TB is a major health problem but leprosy prevalence has declined considerably. The prevalence of leprosy in now falling in many parts of the world, and the future of maintaining existing leprosy projects will be under discussion. The incidence and prevalence of TB remains high.

In the past TB programmes have been chronically underfunded-it was a curable disease not being cured! Recently renewed interest is being given partly because of the link TB has with AIDS. WHO declared TB a 'global emergency' in $1993 .{ }^{4}$ TB treatment has been shown to be one of the most cost-effective health interventions available. ${ }^{5}$

Saunderson ${ }^{6}$ has drawn attention to some of the similarities between leprosy and TB control, as summarized in the section below. 


\section{Similarities between TB and leprosy}

Bacteriology — both acid-fast bacilli spread mainly by droplets from the respiratory tract and necessitating similar diagnostic microscopy facilities.

Treatment and case-finding-Case-finding mainly by encouraging self-reporting through dissemination of information. Case-holding a priority to prevent relapse and treatment failure.

Management and cohort reporting - both rely on the same principles.

Psychosocial/economic aspects. Disruption of patients' lives leading to dehabilitation caused by both diseases.

\section{Advantages of combined programmes}

There are disadvantages in running combined leprosy/TB programmes which benefit the control of both diseases. These are listed below.

A leprosy team and clinic infrastructure can be maintained even when leprosy prevalence falls to low levels.

The diagnosing and treatment of TB patients can be done without duplication of physical and manpower resources.

Staff skills and work methods developed in leprosy can be deployed to the advantage of another very needy group of people.

Integration reduces the stigma of leprosy.

Job security and satisfaction among staff increases.

Leprosy case-finding is maintained by continuing to disseminate information about both diseases.

In 1994 the Danish Bangladesh Leprosy Mission (DBLM), who are a large leprosy control programme in northern Bangladesh, adopted TB control into one part of its working area as a pilot programme. The experience has been very positive, and we share our findings as an encouragement to other programmes considering this kind of step.

\section{PROGRAMME DETAILS}

A joint TB/leprosy control programme has existed in Bangladesh since 1976, but in 1993 the Government of Bangladesh committed itself to an ambitious project of leprosy and TB control with financial support from the World Bank and technical support from WHO. The twin aims were:

the elimination of leprosy as a public health problem by the year 2000 , defined as a reduction in prevalence below $1: 10,000$; and

to reduce the incidence of TB until it is no longer a public health problem by diagnosing and treating effectively as many sputum positive TB patients as possible. The immediate objectives in Bangladesh are to increase the cure rate of new sputum smear positive TB from less than $40 \%$ to $85 \%$ and to increase case detection from the present $10 \%$ to over $50 \%$ of the incidence.

In order to maximize resources, the Government signed a memorandum of understanding in 
1994 with a consortium of leprosy control agencies, the Leprosy Co-ordinating Committee (LCC), of which DBLM is member. Under this memorandum, in return for agreeing to operate leprosy control services in defined districts, leprosy NGOs (Non-Government Organizations) receive multidrug therapy (MDT) supplies free from the Government. Further, leprosy NGOs were encouraged to take up TB control as well, and were offered free drugs to enable them to carry this out, providing the NGO undertook to follow Government guidelines for diagnosis and treatment.

DBLM decided in 1994 to begin a pilot TB control programme in one of its areas of operation with the aim of seeing how well the two disease control structures could sit together. Since its beginning the combined leprosy/TB control programme has mushroomed and is now an established part of DBLM.

\section{Details of the leprosy control programme}

DBLM is a large leprosy control project operating in four northern districts of Bangladesh, an area considered to have the highest prevalence of leprosy in the country $(5 / 1000) .^{7}$ The area is divided into 3 'fields', and in one of them, the Thakurgaon field, the control programme has now been operating since 1978. MDT was introduced in 1984. The Thakurgaon field comprises the districts of Thakurgaon and Panchagar with an area of $3214 \mathrm{~km}^{2}$ and a population of $1,723,000$. It is supported by a small hospital of 16 beds in the district town of Thakurgaon (see Table 1).

The number of new leprosy cases found in this field has been increasing each year since the project began (see Figure 1) because of larger number of staff deployed in the area and intensified case-finding activities (planned before the introduction of the TB programme). Mass surveys indicate that the overall (absolute) prevalence rate is now falling as expected (1991: 10/1000, 1995: 3.7/1000). With the increased proportion of paucibacillary patients, abandonment of routine follow-up (surveillance) after release from treatment (RFT) for relapse/reaction and reduction in active case-finding, the leprosy workload of patient care in the area is actually decreasing. It was for these reasons, and because the authors were living in Thakurgaon at the time, this area was chosen to start the TB pilot project.

\section{Details of the TB programme}

TB is a major public health problem in Bangladesh. The prevalence rate is considered to be 2-3/1000 across the country (Dr Liisa Parkkali, personal communication), and our impression

Table 1. Statistical information relating to leprosy control in the Thakurgaon Field of DBLM in 1994 and 1995

\begin{tabular}{lcc}
\hline & 1994 & 1995 \\
\hline New leprosy patients detected & $652\left(\mathrm{MB}^{*} 148\right)$ & 708 (MB 141) \\
Cases on treatment 31 December & 808 & 600 \\
Registered prevalence/10,000 & $4 \cdot 69$ & $3 \cdot 48$ \\
Number of field staff & 26 & 30 \\
Number of peripheral clinics (mostly monthly) & 22 & 21 \\
\hline
\end{tabular}

\footnotetext{
* Multibacillary.
} 


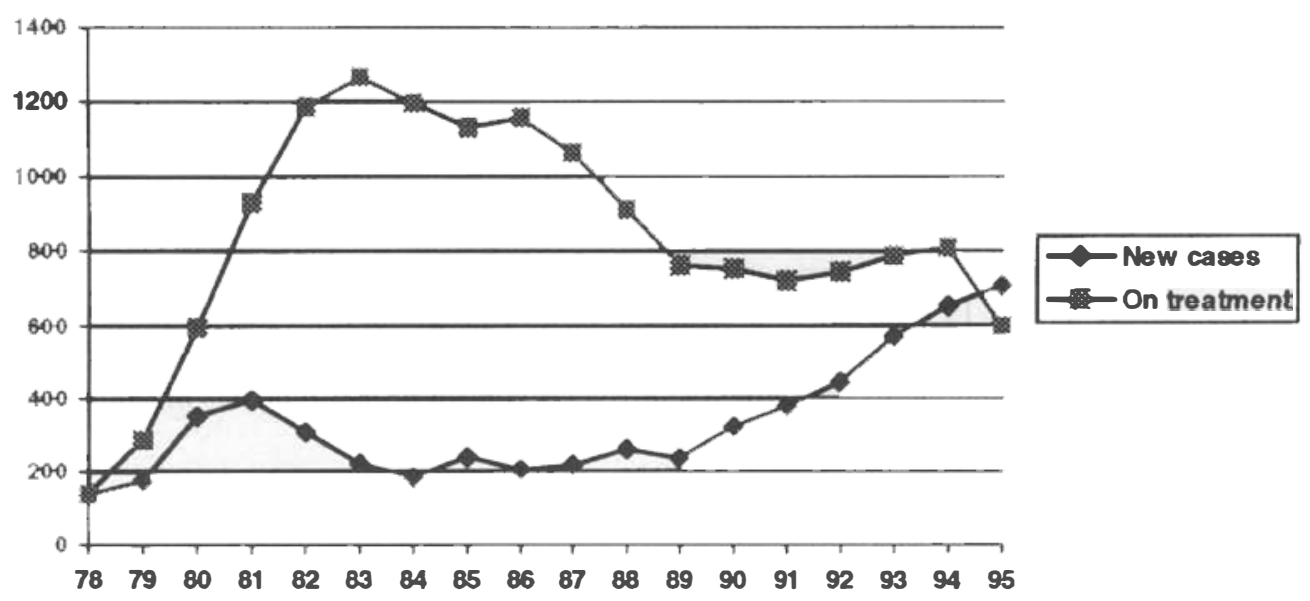

Figure 1. New leprosy case-finding and patients on treatment in DBLM Thakurgaon field, 1978-1995.

is that the level of TB in northern Bangladesh is of that order. Thus in the Thakurgaon project area there is a prevalence of roughly 4500 cases.

The TB programme was started in June 1994 following a short training course for the staff involved. Since then there has been a weekly diagnostic and treatment clinic at Thakurgaon Centre and monthly diagnostic clinics operating at another 4 peripheral clinics. After completing their initial intensive phase of treatment patients can collect their medicines monthly from their nearest leprosy (now leprosy/TB) clinic.

Diagnosis is by sputum examination, and treatment follows the Bangladesh national TB control programme's guidelines. All TB medicines are obtained quarterly from the Government. Since TB work has been started only one additional staff member has been added to the team to help with medicines and record keeping. She packets the TB intensive phase medicines into long cylindrical polythene bags, each day's drugs being sealed into a separate compartment using a candle flame to melt the plastic. The patients are then supplied with one month's medicines, conveniently packed into 28 separate days' supply, as an aid to compliance (the equivalent of MDT blister packs).

All new sputum positive patients receive an 8-month treatment regime consisting of 2 months' intensive phase of daily rifampicin, pyrazinamide, ethambutol and isoniazid followed by a 6-month course of isoniazid and thiacetazone.

Apart from clinic days most of the fieldworkers' time is spent on patient follow up. The Government TB control programme requires that every smear positive TB patient in the first 2-3 months of treatment takes his or her drugs in front of a health worker at least every other day to ensure complete and correct medicine intake. Ideally DOTS- $\underline{\text { Daily }}$ Observed Therapy, Short course-should be implemented to minimize the risk of patient noncompliance and the development of drug resistance. We found this to be operationally difficult with only 1 fieldworker per 60,000 population, so the DBLM field staff enlist the help of a responsible relative who agrees and signs that $\mathrm{s} / \mathrm{he}$ will supervise the patient's medicine intake daily. Compliance is then checked at each weekly visit by the DBLM field worker. It is estimated that excepting clinic time, $60 \%$ of a TB/leprosy field workers' time is taken up with these TB follow up visits, done on a bicycle. 


\section{$T B$ and leprosy case-finding activities}

The case-finding activities have been fully integrated; the fieldworker going to a new area uses a flip chart and gives information to a group of villages about the signs and symptoms of leprosy and TB, telling them what to do if either are suspected. Each weekday night a slide presentation about leprosy and TB is shown in a different village, featuring a series of slides similar to the flip chart; these 'information programmes' are very popular and are often shown to a crowd of over 500 .

In addition to mass information, field staff carry out contact surveys of both leprosy and TB cases. Mass surveys are also part of the leprosy case-finding activities, but this is being reduced steadily because of time constraints. Leprosy case-finding rests increasingly on voluntary self-reporting of cases and this is proving to be successful. In $199570 \%$ of new leprosy cases self-reported, and overall case-finding was higher than 1994.

Leprosy case-finding has not decreased but rather has increased since the addition of the TB programme (see Table 2). Ten leprosy patients have incidentally been found among people attending the TB clinics. The workers have 'leprosy eyes', and suspicious skin patches are sometimes spotted on exposed skin or as the shirt/blouse is lifted for chest auscultation. New leprosy patients have also been found when the fieldworker visits a new area for TB patient follow up. In one case, a fieldworker visited an area where he assumed there was no leprosy. After attending a TB patient in that area he sat with a group, showed his flip chart, and found 5 new cases of leprosy that day.

Unlike leprosy patients, it has usually not been difficult to motivate suspected TB sufferers to attend a clinic. People in Bangladesh are aware that TB is a potentially fatal disease, and it does not carry the stigma that leprosy does. TB clinics have been flooded with people suffering from chest symptoms wanting to have a sputum test simply as a result of mass information. The number of clinic days has purposely been kept low to limit the number of TB patients receiving treatment because of staffing constraints. It is anticipated that in the future more clinics will be offered to increase coverage of the area.

\section{Stigma}

The TB programme may help to decrease the stigma against leprosy. Each month over 1000 people visit the 'leprosy hospital' and 'leprosy clinics' for TB checks. There have been no problems in admitting sick patients to the 'leprosy hospital'.

Table 2. Statistical information relating to TB control in DBLM Thakurgaon Field $1994 / 1995$

\begin{tabular}{lcc}
\hline & 1994 (Jun-Dec) & 1995 (Jan-Jun) \\
\hline New cases registered & 196 & 279 \\
Smear positive cases among new & $158(81 \%)$ & $215(77 \%)$ \\
Number of chest symptomatics screened & 1555 & 4555 \\
Outcome-completed treatment & $151(77 \%)$ & $222(79 \cdot 8 \%)$ \\
\hline
\end{tabular}




\section{Staff response}

In many ways the addition of the TB project has increased the staff workload and it was anticipated that there would be some resistance. However this has not been the case and staff openly admit that this is because the TB project has brought perceived job security. The fieldworkers were not blind to the decreasing prevalence of leprosy with its obvious implications for their future. Many staff, (if not most), had or have relatives or neighbours with TB and were only too aware of the lack of affordable local treatment. The knowledge that their work is worthwhile in reducing death and suffering has of course increased their job satisfaction, and this has been expressed by many of them.

Before working with DBLM one author (RAC) was attached to a TB project running from a general mission hospital. The expertise that the leprosy fieldworkers have brought to the TB work, lacking in those fieldworkers from the general hospital, is very evident. This is especially true in the field of health education, information dissemination and in case holding. The longer-standing fieldworkers also bring to the work a knowledge of the area and the local community whose influence can be invaluable to persuade an infectious defaulting patient to take his medicines.

\section{Financial implications}

Financial implications for DBLM has been minimal since:

TB medicines have been given by the Government; the necessary laboratory equipment and technicians were already in place.

the clinic infrastructure and staff were already in place. An extension has been added to one clinic facility because of the large number of people attending each week;

The hospital infrastructure and staff were in place. Approximately 2-3\% patients require admission for complications, severe illness, drug reactions etc.; and

only one new member of staff has been added to the team specifically to look after the TB records and to help package medicines.

\section{Socioeconomic impressions}

Many TB patients are among the poorest of the poor. An experienced nurse at Thakurgaon hospital commented that the TB patients admitted were worse off than leprosy patients admitted to the same hospital. We conducted a small survey among 21 of our patients registered serially during one month, looking at expenditure and loss of income directly related to their illness before coming to clinic (see Figure 2). Out of the 21 patients interviewed, 11 had lost between Bangladesh Taka (BDT) 8000-40,000 (US\$200-1000), mainly because of loss of income and drug costs. Such sums of money are a huge drain on poor families (average monthly family income in rural Bangladesh is BDT 2670 (US\$65) ${ }^{8}$ ). Often the money is raised by selling livestock and/or land thus impoverishing the family for a long time to come. Eight patients suffered minimal loss (less than BDT 2000-US\$50); 5 of these were able to continue working despite their illness. The mean period from onset of illness until diagnosis at our clinic was 16 months (range 0-60 months). A local synonym for TB is 'King's disease' as it is believed that only kings can afford the treatment and food needed to obtain cure!

Leprosy work has a long history of devoted help for humanitarian or religious reasons to 


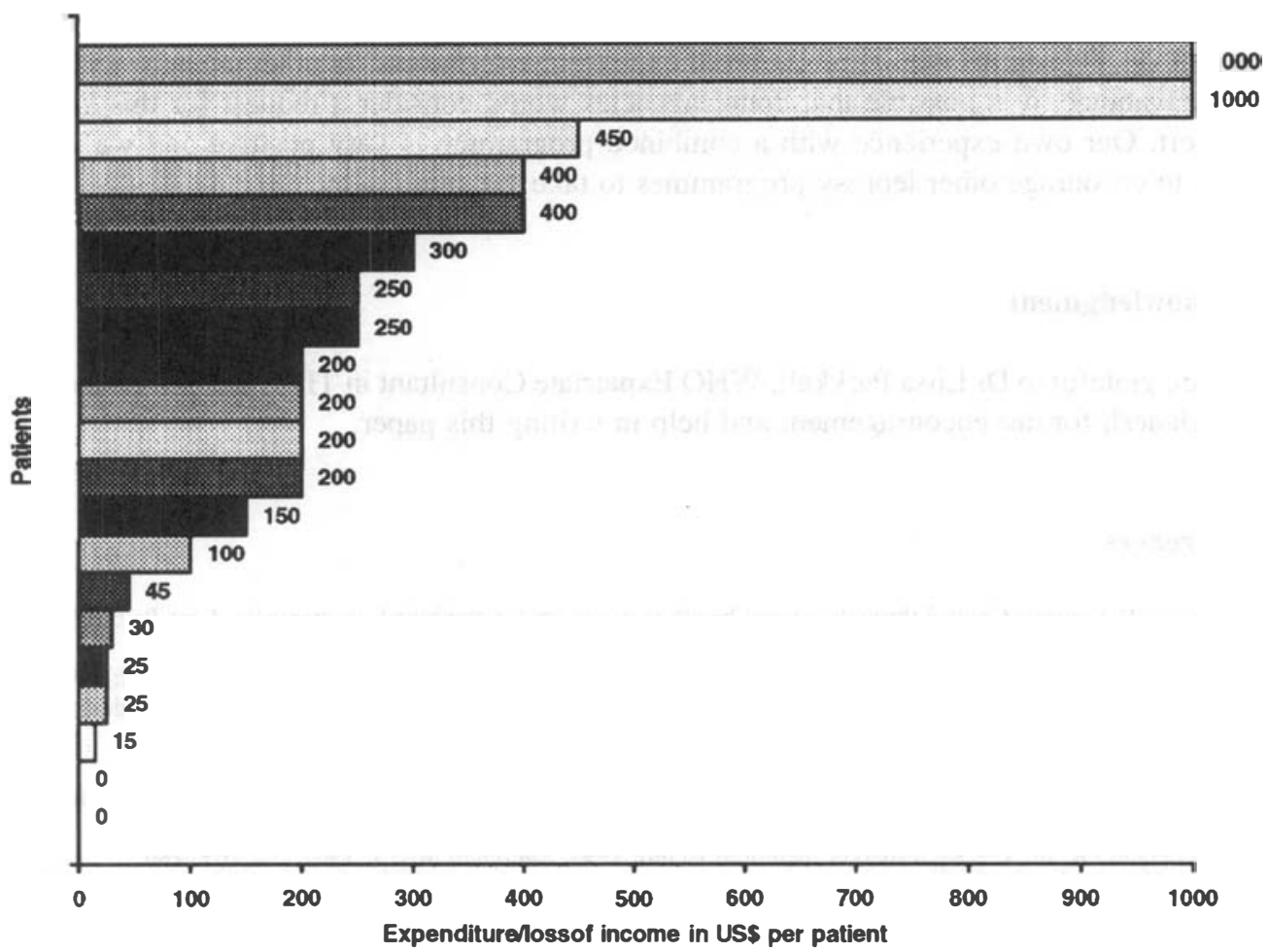

Figure 2. Total loss of income and expenditure related to TB in patients before registration at DBLM TB clinic.

those considered to be at the bottom of the social heap. TB sufferers in developing countries are also in desperate need. While in Bangladesh most do not suffer social stigmatization (only 8 out of 21 in the above study said that their neighbours now avoided their family for fear of catching the disease), in Africa stigma is growing because of the connection with AIDS.

\section{Discussion}

One aim of TB control work is to integrate treatment services into the general health sector, but this is practically very difficult in many developing countries. In the Kellersberger Memorial lecture of 1982, Dr Styblo said that 'in many developing countries TB has been so perfectly integrated that nobody cares about it' ${ }^{9}$ Integrating with another vertical programme, such as leprosy, has much to commend it. It is possible that leprosy control projects are better able to handle the new challenges of TB control than other types of health programme. It is also true that TB control programmes have much to learn from the longer and better established leprosy programmes. ${ }^{3}$ As already stated, it has certainly been our impression that the leprosy workers brought their considerable field experience to bear very positively in the TB work. In our own programme, adding TB control did not bring the feared 'swamping' of leprosy work but in fact injected a new lease of life into it.

It is true that the Bangladesh situation is perhaps better than many, with the Government 
encouraging NGO cooperation and bearing the major cost, that of drugs. This enabled DBLM to pick up TB control with a minimum of expense. Nevertheless, in other situations without this advantage, it is possible that donor agencies would consider a request for this kind of support. Our own experience with a combined programme is very positive and we would want to encourage other leprosy programmes to take the same step.

\section{Acknowledgment}

We are grateful to Dr Liisa Parkkali, WHO Expatriate Consultant in TB to the Government of Bangladesh for her encouragement and help in writing this paper.

\section{References}

${ }^{1}$ Feenstra P. Leprosy Control through general health services and/or combined programmes. Lepr Rev, 1993; 64: 89-96.

${ }^{2}$ Feenstra P. Sustainability of Leprosy Control Services in low-endemic situations. Int J Lepr, 1994; 62: 599-608.

${ }^{3}$ Lockwood DNJ, Saunderson PR. Harnessing the strengths of the leprosy programme to control tuberculosis. $B M J$, 1995; 311: 862-3.

4 TB-a global emergency: WHO report on TB epidemic, WHO 1994.

${ }^{5}$ Murray C, Styblo K, Rouillon A. In Health sector priorities review-tuberculosis. Population, health and nutrition division, The World Bank, Washington DC, June 1991.

6 Saunderson PR. In: The Kellesberger memorial lecture 1994, Ethiopian Med J, 1994; 32: 269-280.

7 Richardus JH, Croft RP. Estimating the size of the leprosy problem: the Bangladesh experience. Lepr Rev, 1995; 66: $158-164$

81994 Statistical Yearbook of Bangladesh, Bangladesh Bureau of Statistics, August 1995.

9 Styblo K. The Kellersberger Memorial lecture, 1982 Ethiopian Med J, 1993; 21: 101 (quoted by Saunderson P, in: The Kellersberger Memorial lecture, 1994 Ethiopian Med J, 1994; 32: 269-280). 